\title{
High-power EUV lithography: spectral purity and imaging performance
}

\author{
Mark van de Kerkhof, ${ }^{a}{ }^{*}$ Fei Liu, ${ }^{\text {a }}$ Marieke Meeuwissen, ${ }^{\text {a }}$ Xueqing Zhang, \\ Muharrem Bayraktar, ${ }^{b}$ Robert de Kruif, ${ }^{a}$ and Natalia Davydova ${ }^{a}$ \\ ${ }^{a}$ ASML Netherlands B.V., Veldhoven, The Netherlands \\ ${ }^{b}$ University of Twente, Industrial Focus Group XUV Optics, Enschede, The Netherlands
}

\begin{abstract}
With the introduction of the NXE:3400B scanner, ASML has brought extreme ultraviolet lithography (EUV) to high-volume manufacturing (HVM). The high-EUV power of $>200 \mathrm{~W}$ being realized with this system satisfies the throughput requirements of HVM, but also requires reconsideration of the imaging aspects of spectral purity, both from the details of the EUV emission spectrum and from the deep-ultraviolet (DUV) emission. We present simulation and experimental results for the spectral purity of high-power EUV systems and the imaging impact of this, both for the case of with and without a pellicle. Also, possible controls for spectral purity will be discussed, and an innovative method will be described to measure imaging impact of varying conversion efficiency (CE) and DUV. It will be shown that CE optimization toward higher source power leads to reduction in relative DUV content, and the small deltas in EUV source spectrum for higher power do not influence imaging. It will also be shown that resulting variations in DUV do not affect imaging performance significantly, provided that a suitable reticle black border is used. In summary, spectral purity performance is found to enable current and upcoming nodes of EUV lithography and to not be a bottleneck for further increasing power of EUV systems to well above 250 W. (C) 2020 Society of Photo-Optical Instrumentation Engineers (SPIE) [DOI: 10.1117/1.JMM.19.3.033801]
\end{abstract}

Keywords: extreme ultraviolet lithography; high power; out-of-band; deep ultraviolet; spectral purity; pellicle; dynamic gas lock membrane.

Paper 20031 received Jun. 23, 2020; accepted for publication Sep. 4, 2020; published online Sep. 22, 2020.

\section{Introduction}

As state-of-the-art high-volume manufacturing (HVM) shifts to 7- and 5-nm nodes, extreme ultraviolet lithography (EUV) has now become fully accepted as the technology of choice. ${ }^{1}$ With the increasing EUV source power and resist improvements, it is time to re-evaluate the spectral purity in terms of imaging impact.

The laser-produced plasma (LPP), as used in the ASML EUV source, emits a broadband spectrum. This spectrum can be approximated as Planck-like blackbody radiation, but the details will depend on the plasma parameters, which are determined by source operating conditions. In general, out-of-band (OOB) EUV (outside of 13.2 to $13.8 \mathrm{~nm}$ ) is undesirable since it might cause mirror heating and lifetime issues in the illuminator. Further downstream this OOB EUV will be step-by-step reduced by the repeated selective reflectivity of the consecutive narrowband Braggtype multilayer (ML) mirrors, so this is not expected to be an issue at wafer level. The vacuumultraviolet (VUV) component between 70 and $110 \mathrm{~nm}$ is effectively removed by the background hydrogen gas; ${ }^{2}$ however, this suppression becomes less effective above $110 \mathrm{~nm}$. The ML mirrors mitigate the deep-ultraviolet (DUV) component, especially in the range between 110 and $200 \mathrm{~nm}$, as discussed in Sec. 3. Figure 1 shows the $\mathrm{H}_{2}$ photoionization and photodissociation cross section from 50 to $115 \mathrm{~nm}$. Furthermore, typical photoresists at wafer level are based on organic molecules that are intrinsically sensitive to DUV light from 150 to $300 \mathrm{~nm}$, resulting in possible contrast loss. ${ }^{3}$ This imposes strict requirements on the DUV/EUV ratio in resist at wafer

*Address all correspondence to Mark van de Kerkhof, E-mail: mark.van.de.kerkhof-msd@asml.com

$1932-5150 / 2020 / \$ 28.00$ CC 2020 SPIE 


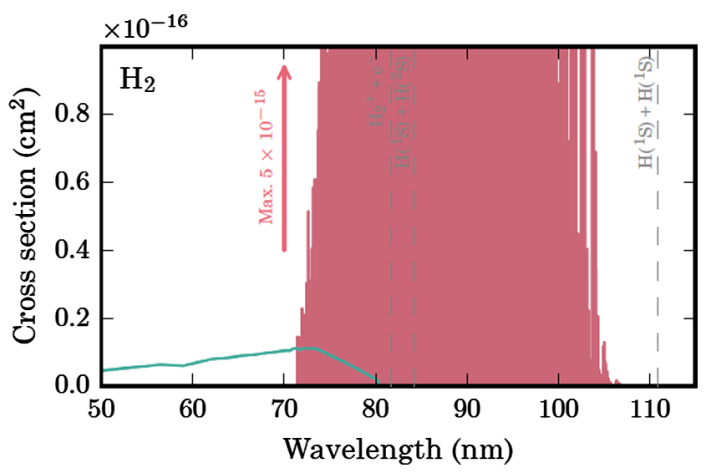

Fig. 1 Cross sections of $\mathrm{H}_{2}$ molecules and absorption zones beneath $110 \mathrm{~nm}$. Red: photodissociation and blue: photoionization. Reproduced with permission from Heays et al. ${ }^{5}$

level for EUV scanners. Especially, DUV reflected from reticle black border (BB) and/or reticlemasking blades can overexpose edges and corners of adjacent fields. EUV pellicles have significantly higher DUV reflection and thus have more impact on CD at edges and corners; to mitigate this, a DUV-suppressing dynamic gas lock membrane (DGLm) has been introduced as an optional upgrade to NXE:3400 to suppress DUV at wafer level. ${ }^{4}$

This paper provides an overview of the spectral purity performance of high-power EUV systems, including discussions on the measurements and simulations results of source spectra, BBs, optics coatings, pellicle, and the resulting imaging performance. Section 2 summarizes the EUV spectra measurement results on ASML EUV sources under different operating conditions and rigorous imaging simulation results of using different measured EUV spectra. Section 3 discusses source DUV spectra measurement results, BB considerations, and optics coatings. Section 4 presents imaging qualification results with and without pellicle and with and without DGLm. The conclusions are given in Sec. 5.

\section{Source Plasma EUV Emission Spectra}

The LPP EUV source intrinsically emits a broadband spectrum. The detailed source spectrum is highly relevant for the source-scanner integral system performance. High-power LPP systems achieve high conversion efficiency (CE) using two closely timed IR-laser pulses per EUV pulse, to optimize EUV emission from the plasma. ${ }^{6}$ The high-level LPP EUV source operation scheme is shown in Fig. 2.

The current generation of LPP EUV sources uses a prepulse (PP) laser focused onto a stream of liquid Sn droplets. The interaction of the PP laser with each tin droplet causes the droplet to deform into a disk-like target with a reduced thickness that is more favorable for EUV production and has a reduced self-absorption. As the tin target is formed, a high-power ( $>20 \mathrm{~kW}), 10.6-\mu \mathrm{m}$

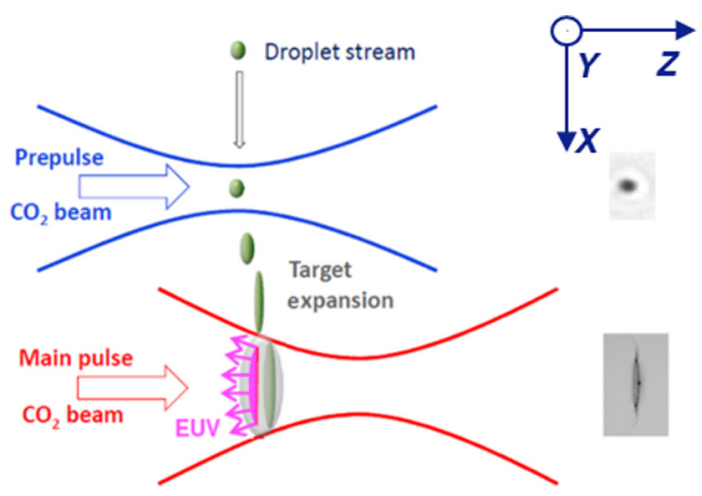

Fig. 2 Principle of split-pulse scheme of high-power LPP EUV source. 


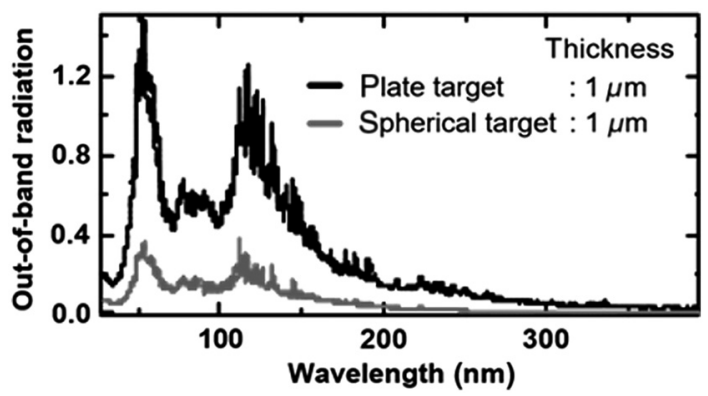

Fig. 3 Full-spectrum OOB measurement by Sakaguchi et al., comparing extended Sn plate target to mass-limited Sn droplet target, showing $\sim 4 \times$ less OOB radiation for mass-limited droplet. ${ }^{10}$ This also shows photodissociation/ionization gap below $120 \mathrm{~nm}$.

wavelength short-pulse $\mathrm{CO}_{2}$ laser main pulse (MP) beam is used to rapidly heat and ionize the disk-like tin target. An intensely hot plasma with a temperature of several tens of eV is generated, and it efficiently emits EUV radiation, in accordance with Wien's classical law ${ }^{7}$ for blackbody radiation:

$$
\lambda_{\max }(\mathrm{nm}) \approx 3.10^{6} / T_{e} \approx 250 / T_{e},
$$

which corresponds to a plasma electron temperature of $\sim 20 \mathrm{eV}$ for $\lambda_{\max }=13.5 \mathrm{~nm} .^{8}$ In practice, this approximation turns out to be a lower boundary, and LPP EUV production at $\lambda=13.5 \mathrm{~nm}$ was found to be most efficient at $T_{e}=30$ to $100 \mathrm{eV}$ by Fomenkov. ${ }^{9}$

In addition to EUV, VUV and DUV are also emitted as blackbody radiation, especially from the slightly cooler periphery of the Sn plasma, and during cooling-down of the plasma, ${ }^{10}$ as shown in Fig. 3. A more uniform Sn plasma, by optimized PP, will help to reduce the energy in these unwanted wavelength bands and concentrate energy in the EUV wavelength band. Alternatively, a mass-limited target, such as smaller Sn droplets, will also help to reduce the energy in the OOB radiation, although this should be balanced again absolute EUV power and droplet stability.

Extensive source operating condition scans were performed on an ASML proto EUV machine to investigate the plasma EUV emission spectrum sensitivities. The PP laser focus to droplet distance along the beam propagation $Z$ axis [laser-to-droplet distance (L2Dz)] was identified as the most effective source control knob to fine-tune the EUV full-band (FB) (13.2 to $13.8 \mathrm{~nm}$ ) spectrum. By scanning the PP focus to droplet distance in $Z$ direction, the MP beam size on Sn target is changed. The normalized EUV FB spectrum shows a sensitivity to MP fluence on Sn target: with constant MP energy and larger MP beam size, EUV emission tilts toward longer wavelengths, indicating underheating of the Sn plasma; the main emission feature from 13.2 to $13.8 \mathrm{~nm}$, however, is quite stable against variations in laser fluence around the baseline working point of source operating conditions. ${ }^{11}$ Figure 4(a) shows the spectra measured from 10 to $60 \mathrm{~nm}$. Two sets of data that were acquired under the same source operation condition but at a different time are plotted to show the good reproducibility. The spectrometer is accurate in the range of interest around $13.5 \mathrm{~nm}$, but above $25 \mathrm{~nm}$, the measured spectrum is disturbed by the high diffraction orders from the main peak around $13.5 \mathrm{~nm}$; for this spectral range ( 25 to $60 \mathrm{~nm}$ ), novel measurement techniques are currently being investigated. Figure 4(b) shows the EUV spectra comparison between a $125-\mathrm{W}$ source and a $250-\mathrm{W}$ source, zooming in on the 7 - to 16-nm range. The higher power source has a higher spectral purity above $13.5 \mathrm{~nm}$ due to better MP absorption by the Sn target and higher MP power density.

\subsection{Impact of EUV Spectrum Variation on Imaging}

To assess the predicted impact of EUV spectral stability also for selected imaging use cases of future nodes on high-NA scanner systems, simulations have been performed with Panoramic Hyperlith. These simulations contain all available scanner projection properties, such as aberrations, Jones pupils (including apodization, diattenuation, and all polarization-dependent phase 


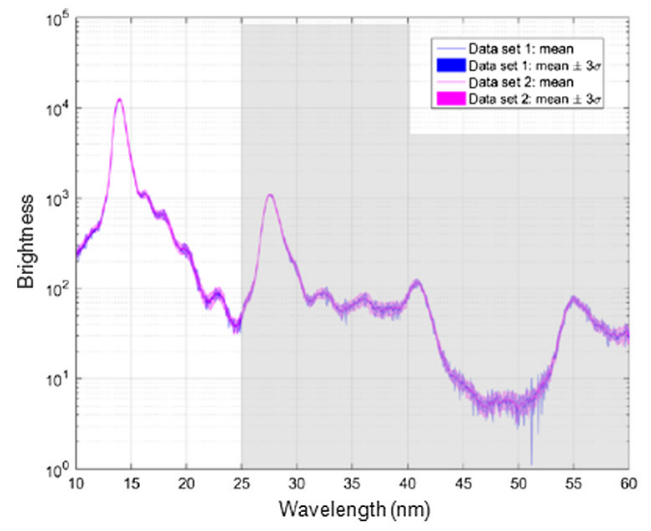

(a)

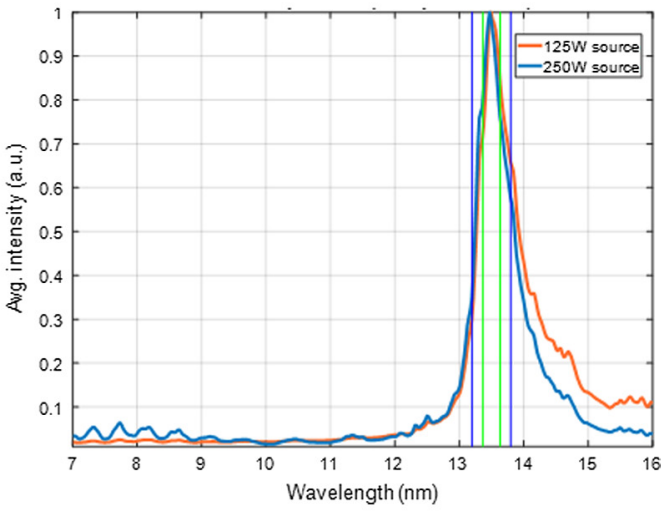

(b)

Fig. 4 (a) EUV spectrum as measured, showing major measurement artifacts at higher diffraction orders for $\lambda>25 \mathrm{~nm}$, making that part of the spectrum unmeasurable by simple grating technique. (b) Zoom-in on 13.5-nm region of EUV spectra from 125- and 250-W source; $250-\mathrm{W}$ source clearly shows better spectral purity.

effects), MSD, and resist blur $(2.5 \mathrm{~nm})$. The proposed high-NA mask stack is defined as a capped Mo-Si ML with 55.3-nm Ta absorber (41.3 nm TaBN and $14 \mathrm{~nm} \mathrm{TaBO}$ ). Wavelength-dependent Jones pupils are used and the wavelength together with the $n$ and $k$ values of the ML and absorber materials are varied in the simulation package. The $n$ and $k$ values used for all materials were taken from the online CXRO database ${ }^{12}$ and Davydova et al. ${ }^{13}$ For each wavelength of the EUV spectrum, the stack parameters are adapted to the correct value and the scanner Jones pupils are updated. Then a wavelength-dependent diffraction pattern was calculated, which results in a wavelength-dependent subimage. The subimages are summed according to the spectrum intensity distribution at reticle level. There are two ingredients that will transfer the reticle-level spectrum to the wafer-level spectrum by the simulation. First, the diffraction simulation from the reticle takes into account how the strength and phase of the diffraction orders are modified for each wavelength since the diffraction calculation is done for every wavelength separately and the material parameters are updated for each wavelength. Second, the wavelength-dependent Jones pupils describe how the reflectivity of the lenses depends on wavelength, and in this way the strength and phase of the diffracted waves are modified to finally obtain the spectrum at wafer level. In this manner, the EUV wafer-level spectrum is automatically generated by Panoramic Hyperlith. The "image averaging" method from Panoramic Hyperlith is used, where the resulting image (wafer level) is calculated as the average of subimages that are obtained for a range of wavelengths. The impact of spectrum stability on high-NA $(\mathrm{NA}=0.55)$ imaging was investigated by comparing the simulated results of two different measured EUV spectra at plasma shown in Fig. 5(a). It should be noted that while the spectrum is sensitive to targeting conditions at the source output, the EUV spectrum at wafer level always shows a stable spectrum due to the strong spectral filtering by the ML mirrors of the optical column.

The first-source EUV spectrum was acquired at the baseline source operating condition, which is determined by a standardized source optimization procedure, with a PP laser focus to droplet distance in $Z$ direction (L2Dz) at $105 \mu \mathrm{m}$. The second source EUV spectrum was acquired with an enlarged L2Dz setpoint at $505 \mu \mathrm{m}$. As discussed in Sec. 2, the EUV FB is a weak function of the MP fluence. For this particular source condition, by increasing L2Dz to $505 \mu \mathrm{m}$, the MP fluence on Sn target is reduced to a low value of $300 \mathrm{~J} / \mathrm{cm}^{2}$, thus a slightly shifted EUV spectrum is obtained. The spectra at the reticle level (input of the simulation) are obtained by applying a transfer function (which translates the measured source plasma spectrum to the spectrum at reticle level by taking the ML properties of the collector and illuminator mirrors into account) to the two measured source plasma spectra.

The imaging study is carried out on three well-defined use cases. These use cases are listed in Fig. 6. The plots of the illuminations used are shown as well. For each use case, the densest pitch is used as dose anchor. The spectrum-induced changes in imaging key performance 


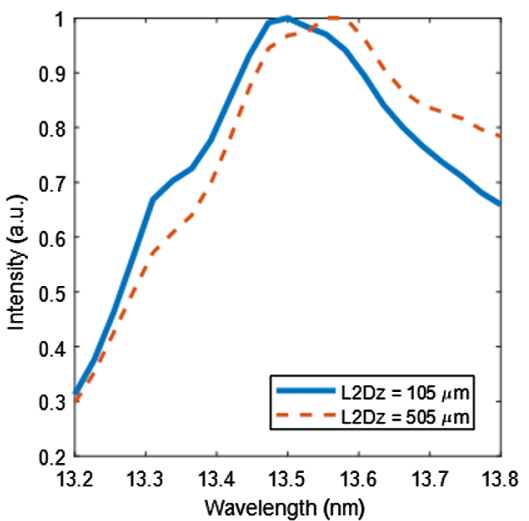

(a)

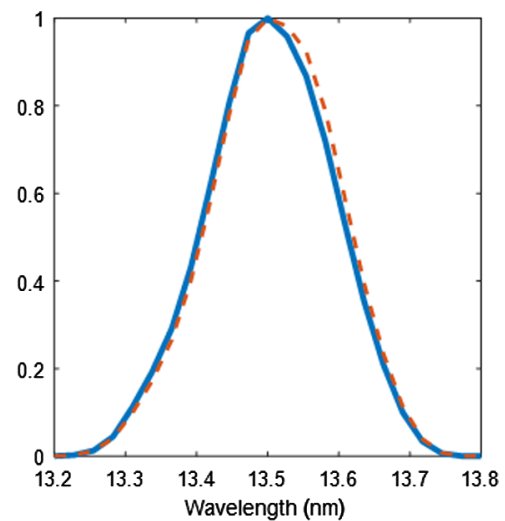

(b)

Fig. 5 EUV FB spectra (two extreme cases): (a) at source and (b) at wafer level, after spectral filtering by the optical column. L2Dz is laser to droplet distance of PP in $Z$ direction.

\begin{tabular}{|c|c|c|c|c|}
\hline & $\begin{array}{l}2 \text { trench } \\
\text { (11/11P110 nm } \\
\text { H,V) Dipole-90 } \\
\text { 38\% PFR }\end{array}$ & $\begin{array}{l}\text { Space through } \\
\text { pitch }(8 \mathrm{~nm} \mathrm{H} \text {, } \\
\text { V) Leaf } \\
\text { Dipole } 20 \% \\
\text { PFR }\end{array}$ & \multicolumn{2}{|c|}{$\begin{array}{l}\text { Pixelized illumination } \\
\text { L/S through pitch }\end{array}$} \\
\hline $\begin{array}{l}\text { Use case } \\
\#\end{array}$ & $\begin{array}{l}\text { UC1H: } \operatorname{Dip} Y \\
\text { UC1V: DipX }\end{array}$ & $\begin{array}{l}\text { UC2: leaf } \\
\operatorname{Dip} Y\end{array}$ & \multicolumn{2}{|l|}{ UC3: pixelized } \\
\hline $\begin{array}{l}\text { Mask } \\
\text { CD/pitch }\end{array}$ & $\begin{array}{l}\text { UC1H: } \\
\text { L11/S11 } \\
\text { P110 H } \\
\text { UC1V: } \\
\text { L11/S11 } \\
\text { P110V }\end{array}$ & $\begin{array}{l}\text { CD8P16H } \\
\text { CD10P20H } \\
\text { CD12P24H } \\
\text { CD14P28H } \\
\text { CD16P40V }\end{array}$ & $\begin{array}{l}\text { UC3H: } \\
\text { CD12P24H } \\
\text { CD12P27H } \\
\text { CD12P30H } \\
\text { CD12P35H } \\
\text { CD12P50H } \\
\text { CD12P80H } \\
\text { L12S12P60H }\end{array}$ & $\begin{array}{l}\text { UC3V: } \\
\text { CD12P24V } \\
\text { CD12P27V } \\
\text { CD12P30V } \\
\text { CD12P35V } \\
\text { CD12P50V } \\
\text { CD12P80V } \\
\text { L12S12P60V }\end{array}$ \\
\hline
\end{tabular}

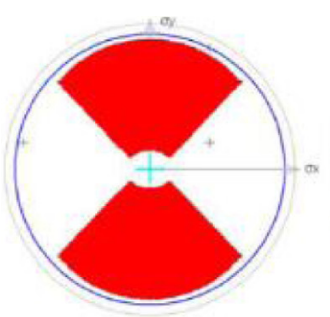

DipY

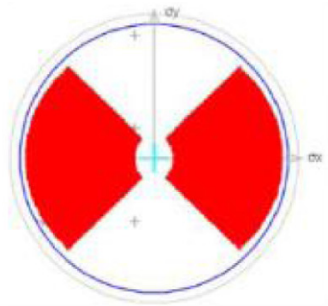

DipX

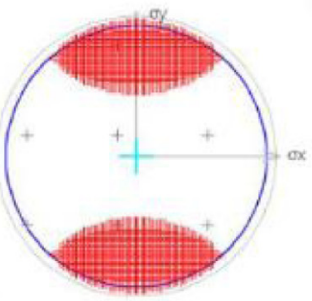

Leaf DipY

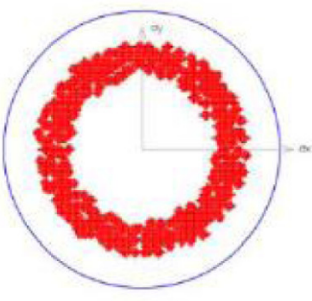

Pixelized annular

Fig. 6 High-NA (NA = 0.55) imaging simulation use cases definitions; notations show CD/biasvalue $C D$ for lines $L$, spaces $S$, and pitch $P$, and orientations $H$ (horizontal) or $V$ (vertical).

indicators (KPI's), e.g., critical dimension ( $\Delta \mathrm{CD})$, normalized image log slope ( $\Delta$ NILS), and pattern placement error ( $\triangle \mathrm{PPE})$, are summarized in Table 1 . All $\Delta$-values are very small. The largest $\Delta \mathrm{CD}, \Delta \mathrm{NILS}$, and $\Delta \mathrm{PPE}$ are 0.043 (UC3), 0.016 (UC2H), and $0.054 \mathrm{~nm}$ (UC2H), respectively, as shown in Table 1. Therefore, the impact of the EUV spectral stability on imaging is small in the total budget; as is to be expected given the homogenizing effect of the mirrors of the optical column, as shown in Fig. 5(b). 
Table 1 Summary of the changes of KPIs ( $\triangle C D, \triangle N I L S$, and $\triangle P P E)$ due to spectrum stability. Focus range is -20 to $20 \mathrm{~nm}$.

\begin{tabular}{|c|c|c|c|c|}
\hline $\begin{array}{l}\text { Use cases } \\
\text { (UC) }\end{array}$ & $\underset{(\mathrm{nm})}{\operatorname{Maximum}} \Delta \mathrm{CD}$ & Maximum $\Delta$ NILS & $\underset{(\mathrm{nm})}{\operatorname{Maximum}} \Delta \mathrm{PPE}$ & Details \\
\hline UC1H & 0.027 & 0.009 & 0.022 & Two-bar, Dip $Y$ \\
\hline UC1V & 0.031 & 0.011 & 0.011 & Two-bar, Dip $X$ \\
\hline UC2 & 0.034 & $\begin{array}{l}0.016 \text { (at }-20 \mathrm{~nm} \\
\text { defocus) }\end{array}$ & $\begin{array}{c}0.054 \text { (at } 20 \mathrm{~nm} \\
\text { defocus) }\end{array}$ & $\begin{array}{l}\text { Spaces through pitch, } \\
\text { Dip } Y\end{array}$ \\
\hline $\mathrm{UC3H}$ & 0.035 & 0.010 & 0.001 & Pixelized through pitch, $\mathrm{H}$ \\
\hline UC3H & $\begin{array}{l}0.043 \text { (at }-20 \mathrm{~nm} \\
\text { defocus) }\end{array}$ & 0.012 & 0.005 & Pixelized, two-bar, H \\
\hline UC3V & 0.040 & 0.011 & 0.001 & Pixelized through pitch, $\mathrm{V}$ \\
\hline UC3V & 0.012 & 0.008 & 0.001 & Pixelized, two-bar, V \\
\hline
\end{tabular}

Note: The largest changes for each KPI are highlighted in bold.

\section{Source Plasma DUV Emission Spectra}

The DUV spectrum from the LPP source is a function of the source operating conditions. The spectra were measured from a metrology port orthogonal to the drive laser axis using a broadband transmission grating spectrometer developed by University of Twente. ${ }^{14}$ For the DUV spectra measurements, the CCD camera integration time was set to $0.5 \mathrm{~s}$, and therefore each spectrum is the result of averaging $\sim 25,000$ EUV pulses, given the 50-kHz frequency of the LPP source. The detailed spectral measurement setup is shown in Fig. 7. Figure 8 shows DUV spectra comparison between a $125-\mathrm{W}$ source and a $250-\mathrm{W}$ source at wafer level, derived from measurement directly at Sn plasma. It should be noted that compared to $125-\mathrm{W}$ source, $250-\mathrm{W}$ source uses higher peak power MP to increase CE, which results in a slightly reduced DUV/EUV ratio.

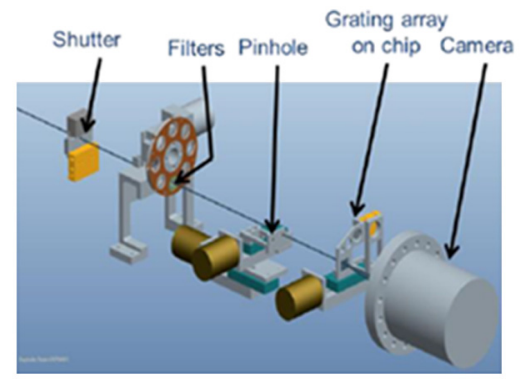

(a)

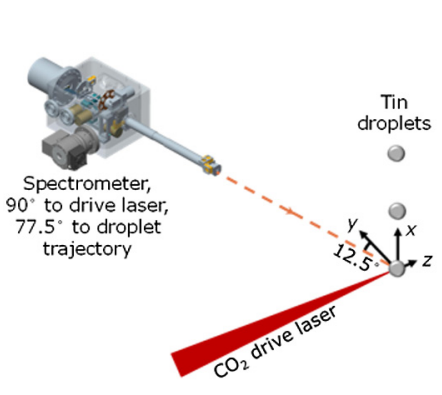

(b)

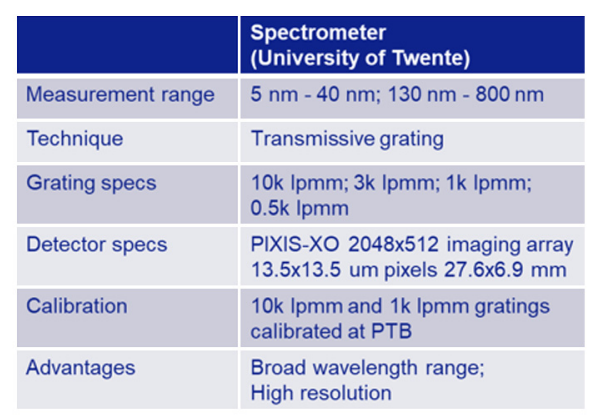

(c)

Fig. 7 (a) Key components of EUV transmission grating spectrometer, developed at University of Twente and (b) schematics of spectra measurement setup. (c) Spectrometer specifications sheet from Refs. 17 and 18. 


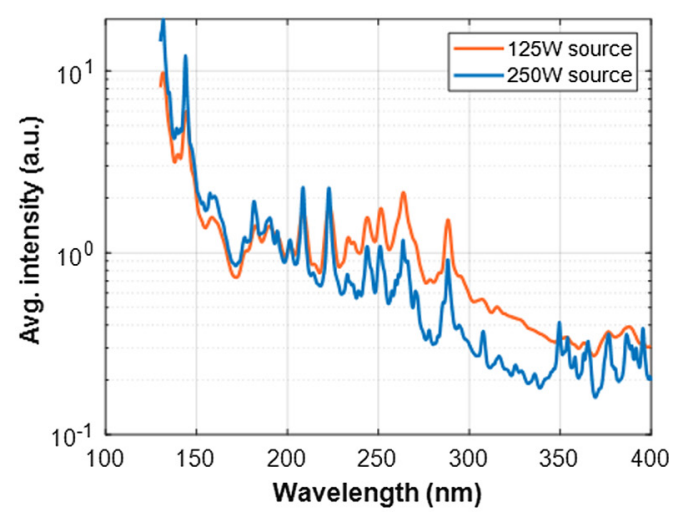

(a)

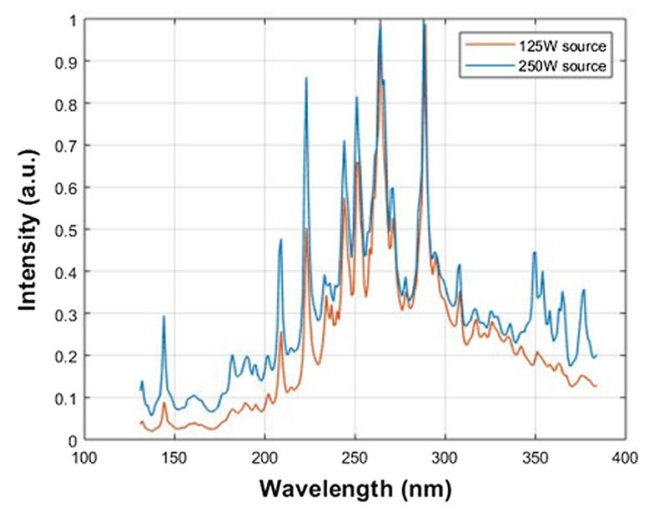

(b)

Fig. 8 (a) DUV spectra directly measured from Sn plasma and normalized to the EUV power; ratio DUV/EUV is roughly the same for both 125 and $250 \mathrm{~W}$. (b) DUV spectra from 125- and 250-W source at wafer level in vacuum. It is worthy to note that the wafer-level spectra are derived from measurement at plasma and have been normalized to its peak, respectively.

The exact dependencies of DUV emission on droplet sizes, PP effects, MP energy, duration, and peak power are currently being experimentally investigated for potential of further reduction. ${ }^{15}$ DUV emission lines are mainly identified from the lower ionization states of $\mathrm{Sn}: \mathrm{Sn}^{1+}, \mathrm{Sn}^{2+}$, and $\mathrm{Sn}^{3+}$. Properly designed EUV resists are not sensitive to DUV light above $300 \mathrm{~nm},{ }^{16}$ whereas wavelengths below $110 \mathrm{~nm}$ are effectively not transmitted through the hydrogen background gas.

However, since some unknowns remain, such as the DUV emission angular dependency of the plasma, the collector optics reflectivity over this wavelength range, and the wavelengthdependent sensitivity of the photoresist, the final DUV performance qualification is done at wafer level, based on dose-to-clear exposure measurements in resist. In this standardized qualification test, reticles with both ML and chromium areas are exposed through dose to determine the dose-to-clear for EUV and DUV, respectively ${ }^{3}$; $\mathrm{Cr}$ has a high-DUV reflectance of $\sim 60 \%$ for wavelengths above $190 \mathrm{~nm}$ and very low-EUV reflectance $<0.05 \%$. For best accuracy, the exact DUV/EUV ratio in certain type of resist at wafer level should be corrected for the exact DUV spectrum at reticle level (often unknown, since it is source plasma recipe dependent), the effective DUV wavelength-dependent reflectivity of Cr, the EUV reflectivity of the ML, and the wavelength-dependent sensitivity of the photoresist (often unknown). In practice, DUV/EUV ratio in a specific resist is determined and reported from the ratio of dose-to-clear from ML and the dose-to-clear from $\mathrm{Cr}$ as

$$
\mathrm{DUV} / \mathrm{EUV}=\frac{E_{0}^{\mathrm{ML}}}{E_{0}^{\mathrm{Cr}}} \cdot 100 \% .
$$

From the resist height curve, the dose-to-clear is calculated. Example dose-to-clear curves in resist EUVJ-3030, using NXE:3400 platform and YieldStar metrology, are shown in Fig. 9. The ratio of dose-to-clear between the Cr and ML defines the DUV level of the system. The impacts from BBs and optics coatings will be discussed in detail in following sections. It should be noted that the DUV/EUV ratio at wafer level depends also on the absorber materials, which can be relevant in the choice of alternative absorbers, such as high- $k$ or attenuated phase shift mask materials. ${ }^{19}$

\subsection{Black Border Consideration}

The image BB (also referred to as light shield area or border) on a mask is a pattern-free nonreflecting area surrounding the image field, designed to prevent undesirable exposure of the neighboring field on the wafer, as shown in Fig. 10. The regular Ta-based EUV absorber is insufficient for this purpose as it still has up to $2 \%$ EUV reflectance, and $~ 15 \%$ DUV reflectance, which can result in $>1$-nm edge/corner CD-shifts. ${ }^{3}$ 


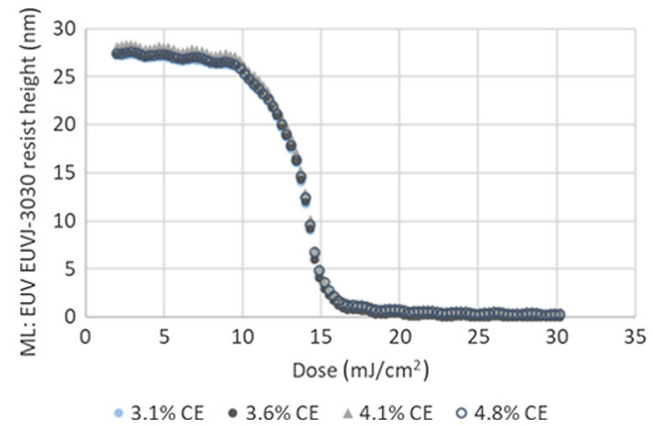

(a)

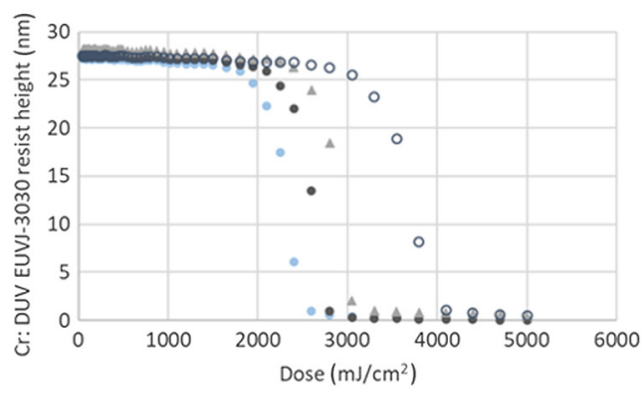

- $3.1 \% \mathrm{CE} \quad \cdot 3.6 \% \mathrm{CE} \quad \triangle 4.1 \% \mathrm{CE} \quad 04.8 \% \mathrm{CE}$

(b)

Fig. 9 Example of dose-to-clear curves of resist EUVJ-3030 using NXE:3400 platform, with left response from $\mathrm{ML}$ (EUV) and right response from $\mathrm{Cr}$ (DUV). The ratio of dose-to-clear between $\mathrm{Cr}$ and $\mathrm{ML}$ gives the DUV level.
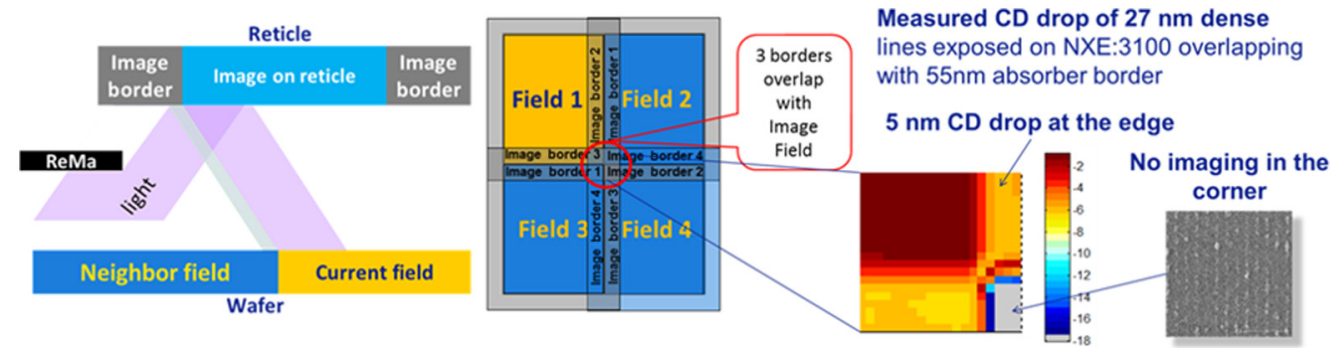

Fig. 10 Illustration of need for nonreflective image border and nonreflective reticle-masking blades to avoid spurious exposure of neighboring fields by EUV and/or DUV. ${ }^{3}$

Several options can be considered to suppress the BB DUV reflectance while simultaneously reducing the EUV reflectance to $<0.1 \%$, for example:

- double absorber,

- etched BB,

- etched and patterned BB, and

- melted BB.

A double absorber layer can meet the EUV reflectance target of $<0.1 \%$ but still has highDUV reflectivity such as the original absorber layer; this can be reduced to $<1 \%$ by patterning the BB area with diffractive grating structures targeted at DUV wavelengths around $200 \mathrm{~nm}$. However, this requires a phase depth of $\sim 100 \mathrm{~nm}$ to create destructive interference for the DUV wavelengths, which complicates the mask manufacturing process significantly; this also has an impact on writing times and cost while the additional coating thickness might result in stresses and pattern shifts at the image edge.

Etched BB gives negligible EUV reflectance from the low thermal expansion material (LTEM) substrate $(<0.02 \%)$ but can still have DUV reflectance of $5 \%$ in the wavelength range between 150 and $300 \mathrm{~nm} .^{20}$ Thus, the etched BB may be combined with additional antireflection-coating or diffractive patterning to further reduce DUV reflectivity to below $1 \%$, as shown in Fig. 11.

A drawback of the etched BB solutions is the risk of PPEs at the field edges, resulting from the ML stress relaxation induced by ML etching, and additional constraints on mask cleaning processes in view of the trench through the ML. The etched trench also results in concerns on defectivity and on the interaction between EUV-induced plasma and the exposed LTEM and the ML sidewall within the trench. ${ }^{21}$

An alternative BB being considered is the "melted" or "heated" BB. Here, the ML is locally (over) heated by a laser, thereby initiating intermixing between the molybdenum and silicon 
van de Kerkhof et al.: High-power EUV lithography: spectral purity...

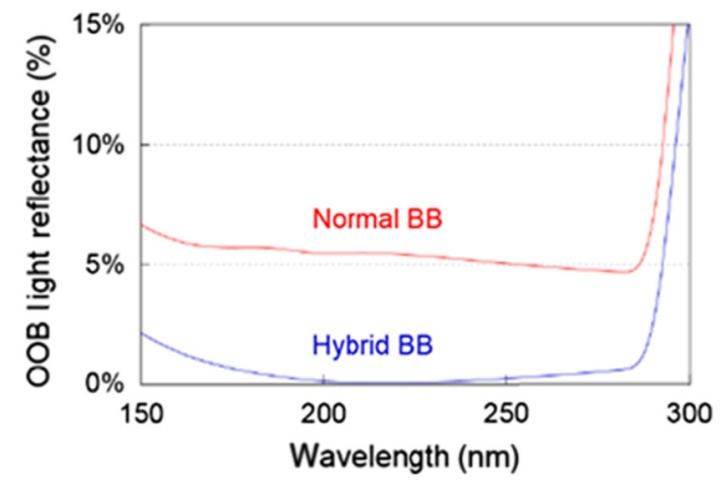

Fig. 11 Etched $\mathrm{BB}^{18}$; red: normal $\mathrm{BB}$, etched down to substrate; blue: hybrid $\mathrm{BB}$, etched plus additional substrate surface structuring and/or treatment.

layers of the mirror ML, resulting in molybdenum-silicide-like layers. These layers have a higher material density and as a result the bilayer thickness, which is a measure for the EUV reflection wavelength, decreases. The reflection wavelength thus shifts to a lower wavelength, away from the optimal transmission wavelength of the projection optics box of the exposure tool. In case of sufficient Mo-Si intermixing, the EUV light will not reach the wafer. It should be noted that, as the double $\mathrm{BB}$, here also the reduction of DUV light is a challenge.

It should be noted that in the near future both the imaging as well as the pattern shift aspects of any chosen BB design will become more critical for the half-field stitching that might be needed for the upcoming anamorphic high-NA EUV platform. ${ }^{22}$

\subsection{Optics Coating}

The illuminator and projection optics of an EUV scanner consist of finely polished reflective mirrors. ${ }^{4}$ Continuous manufacturing optimizations are done to enhance aberrations, EUV transmission, and optics lifetime. In addition to these primary objectives, however, DUV must also be considered to avoid contrast degradation. Figure 12 shows a comparison of DUV performance over systems for both first-generation and current process-of-record coatings, which have enhanced optics lifetime and improved EUV transmission. The average difference in DUV/

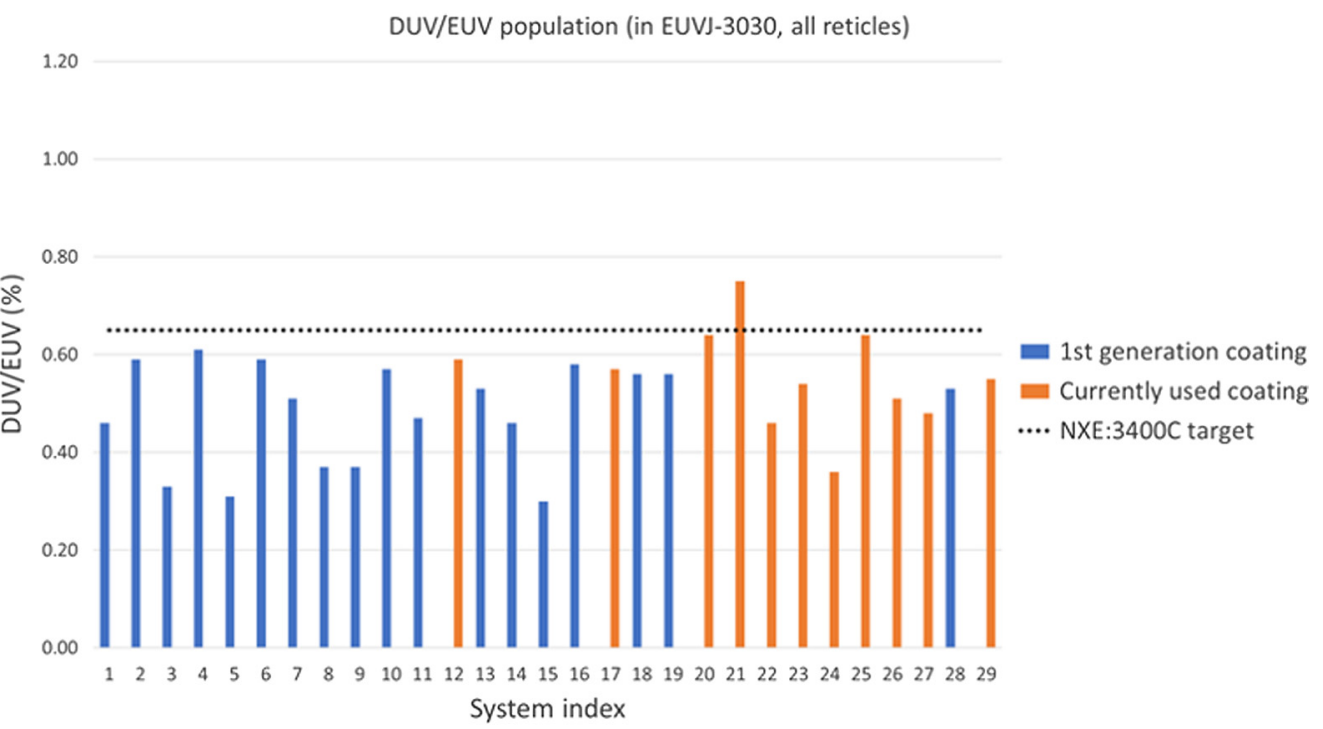

Fig. 12 DUV performance variation over systems, including variations in CE and possible variations in scanner optics; blue indicates first-generation coating, and orange indicates currently used coating; all measured with resist EUVJ-3030. 
EUV ratio between the two types of coatings does not exceed $0.1 \%$. With the newer coatings, on average the DUV/EUV ratio measured in resist increases slightly; however, it is still well within the imaging performance target, as shown in Sec. 4. The variation of performance within the same coating and between different coatings is mainly due to variations in the source CE, as described in more detail in Sec. 4 and Fig. 16, with DUV/EUV ratios of $\sim 0.4 \%$ to $\sim 0.6 \%$ observed as function of CE detuning.

\section{DUV Impact on Imaging}

DUV reflected by image BB from neighboring fields may potentially impact imaging in edges and corners, as shown in Fig. 10.

To highlight the DUV contribution to edge and corner $\triangle \mathrm{CD}$ performance, imaging qualification tests were carried out with and without DGLm, which acts as a spectral purity filter. ${ }^{4}$ With DGLm, DUV reflected by Cr reticle is filtered and cannot expose the resist. As shown in Fig. 13, the DGLm removes all DUV, within measurement limits of the scanner test $(>90 \%)$. Earlier off-line tests at the Physikalisch-Technische Bundesanstalt in Berlin measured $>99 \%$ DUV suppression of DGLm. ${ }^{4}$

In this qualification test, full wafer critical dimension uniformity (FWCDU) wafers were exposed on the same day just before and directly after building in of the DGLm, and the respective intradie fingerprints (corrected for reticle writing errors and shadow-correction) were compared to determine the crosstalk impact of DUV from neighboring fields. The intradie fingerprint results in Fig. 14 show that for both with and without DGLm the corner and edge $\Delta$ CD are all $<0.1 \mathrm{~nm}$, which is well within N5 node requirements. By the introduction of a DGLm this is further reduced to $\sim 0.04 \mathrm{~nm}$, which must be attributed to spurious EUV reflections and flare.

Current EUV pellicles have a higher DUV reflection than BB and thus can have significant impact on $\mathrm{CD}$ at the corners and edges. However, the high-DUV suppression of the DGLm effectively mitigates any CD-impact of DUV reflected from pellicle, as shown by imaging tests shown in Fig. 15. The remaining edge/corner effect of $0.15 \mathrm{~nm}$ with DGLm installed is attributed to the low but finite EUV reflection from pellicle $(<0.04 \%)^{23}$ and is well within N5 node requirements.

Since imaging impact has similar fingerprint, it is not straightforward to separate DUV and EUV contributions to edge/corner effects; this is crucial in order to define proper countermeasures if needed. Comparing with to without DGLm imaging allows such disambiguation but building the required DGLm hardware in or out is a major disruption to the EUV system, so it is considered impractical. As an alternative, we propose a method by detuning of the source CE by varying the L2Dz, as described in Sec. 2. This can be done in-line without opening the system and while running (test) wafers without losing source control.

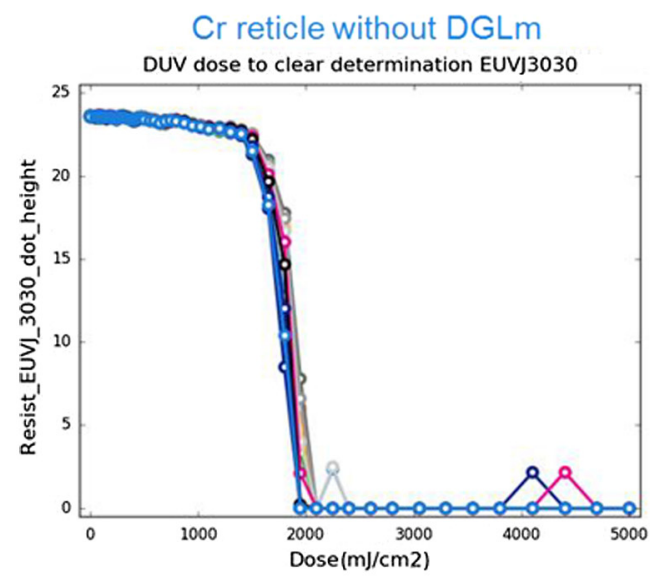

(a)

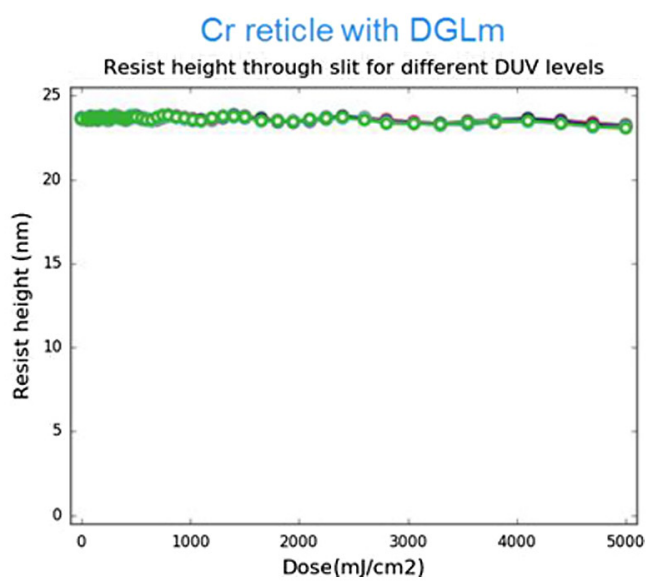

(b)

Fig. 13 Dose-to-clear using a Cr-coated DUV reticle, (a) without and (b) with DGLm. With DGLm, dose-to-clear cannot be determined within limits of test, showing $>90 \%$ reduction of DUV. 


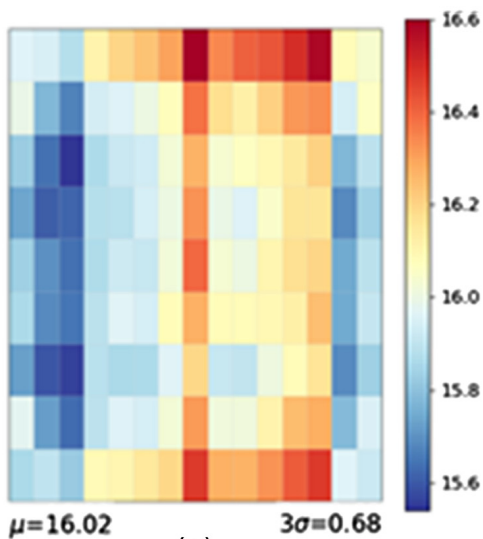

(a)

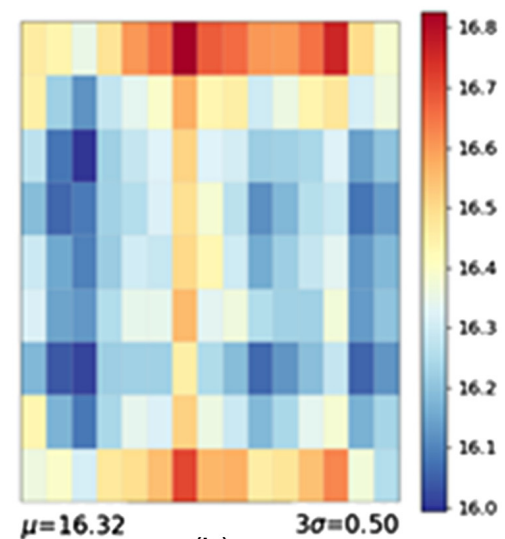

(b)

Fig. 14 Intradie fingerprint of 16-nm DL/spaces, showing almost identical edge/corner effect (a) without and (b) with DGLm. Common fingerprint in $X$ was caused by a suboptimal scanner calibration. Common edge/corner effect is attributed to EUV reflections, whereas delta edge/ corner effect is attributed to DUV; both contributions show $<0.1-\mathrm{nm}$ corner effect.

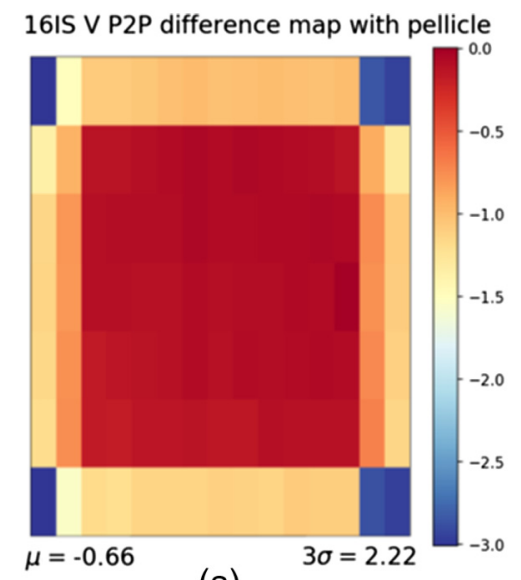

(a)

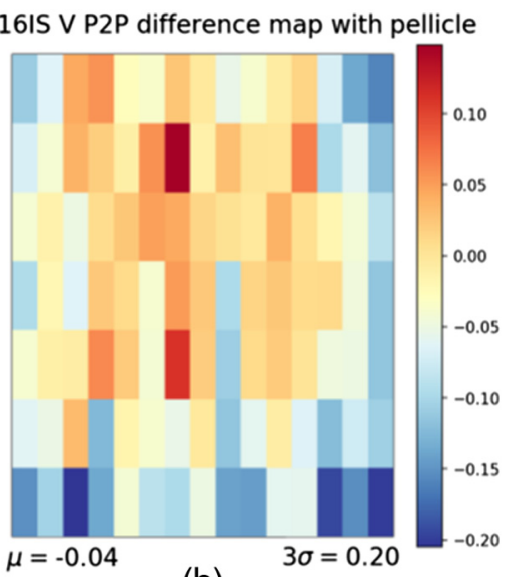

(b)

Fig. 15 Intradie fingerprint showing effective mitigation by DGLm of edge/corner effect of pellicle, for 16-nm IS; (a) without and (b) with DGLm.

The resulting shift in Sn plasma temperature will change the ratio of DUV-to-EUV (also known as OBB), as shown in Fig. 16 for an NXE:3400B (using dose-to-clear for a Cr reticle, as shown in Fig. 9). Assuming that baseline CE is highest possible, in this way 50\% higher DUV-to-EUV ratio can be achieved at wafer level, by change of setpoints alone: the baseline

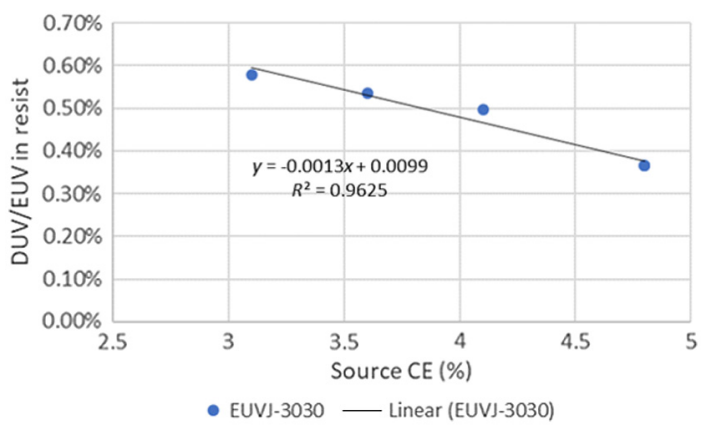

Fig. 16 DUV/EUV ratio measured in resist is changed by detuning CE. 


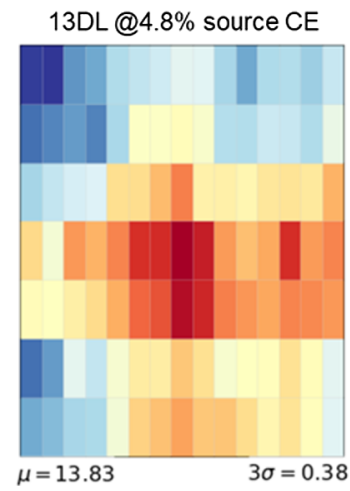

(a)

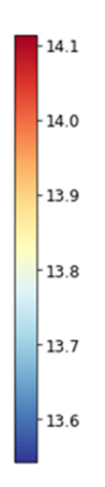

$\mu=13.74$

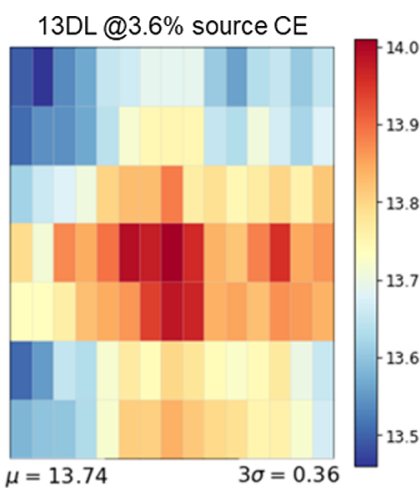

(b)

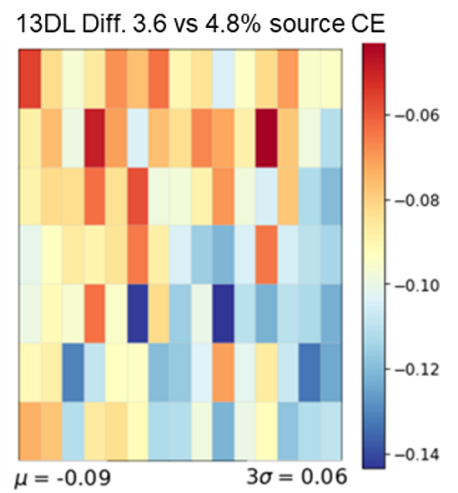

(c)

Fig. $1713-\mathrm{nm}$ DL with abutted fields intrafield CDU in non-CAR metal-oxide resist. (a) Measurement with $4.8 \%$ source CE (low DUV/EUV of $0.37 \%$, as measured in EUVJ3030 ), (b) measurement with $3.6 \%$ source CE (high DUV/EUV of $0.54 \%$, in EUVJ-3030, (c) delta CD map, showing negligible edge/corner impact of varying DUV.

source setting of $\mathrm{CE}=4.8 \%$ corresponds to $\mathrm{OOB}=0.37 \%$ (DUV/EUV ratio as measured in standard EUVJ-3030 test resist), whereas detuned $\mathrm{CE}=3.6 \%$ corresponds to $\mathrm{OOB}=0.54 \%$.

At setpoints for $4.8 \%$ and $3.6 \% \mathrm{CE}$, or OOB levels of $0.37 \%$ and $0.54 \%$, intrafield CDU was measured to establish edge/corner effect, as shown in Figs. 17-20 for 13-nm dense lines (DL). This shows that there are hardly any differences observed in the edges and corners for both higher and lower DUV/EUV ratios, indicating almost no DUV contribution, and almost only limited EUV contribution. For 13-nm DL with abutted fields, the difference plot for higher versus lower DUV is below $0.05 \mathrm{~nm}$ or below the limit of test reproducibility, indicating negligible DUV contribution for this process [using non-chemically amplified resist (CAR) metal-oxide resist]. Comparing abutted to spaced fields in Fig. 19 does show some edge/corner effect, of $0.08 \mathrm{~nm}$ for the corners, which is also close to the limit of the test reproducibility. This is tentatively attributed to the small but nonzero EUV reflectivity of the hybrid BB since Fig. 17 does not show any measurable DUV sensitivity.

At these same OOB levels of $0.37 \%$ and $0.54 \%$, intrafield CDU was also measured to establish edge/corner effect for 16-nm isolated spaces (IS), as shown in Figs. 21-24. This shows that there are again hardly any differences observed in the edges and corners for both higher and lower DUV/EUV ratios, indicating almost no DUV contribution, as well as almost only limited EUV contribution. For 16-nm IS with abutted fields, the difference plot for higher-lower DUV/ EUV is $0.05 \mathrm{~nm}$, which is at the limit of test reproducibility, indicating only minor or negligible DUV contribution for this process (using state-of-the-art CAR resist). Common to both lower and higher DUV/EUV, some additional EUV sensitivity can be observed, of order of $0.1 \mathrm{~nm}$, which is also at the limit of the test reproducibility. Comparing abutted to spaced fields in Fig. 23

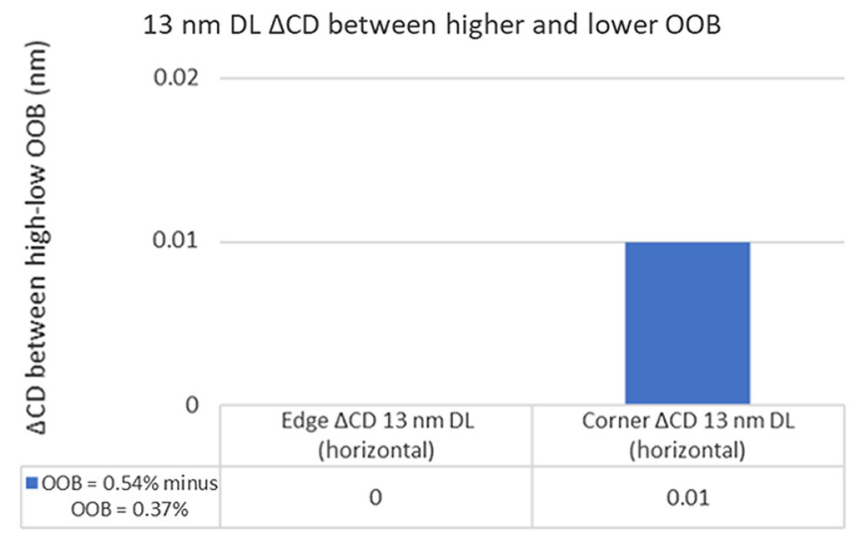

Fig. $18 \mathrm{CD}$-impact on $13 \mathrm{~nm} \mathrm{DL}$ with abutted fields, difference between $\mathrm{OOB}=0.54 \%$ case and $\mathrm{OOB}=0.37 \%$ case. 


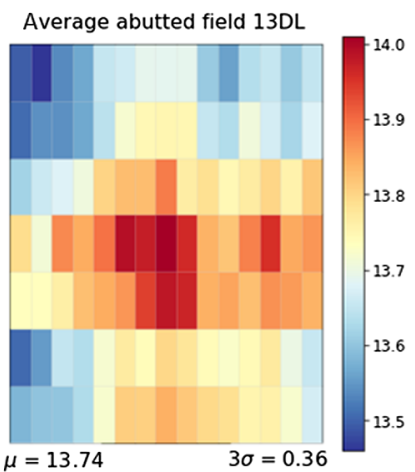

(a)

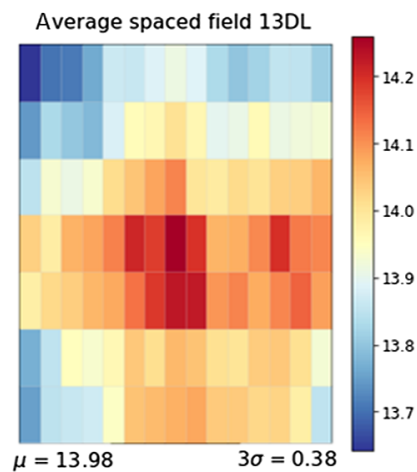

(b)

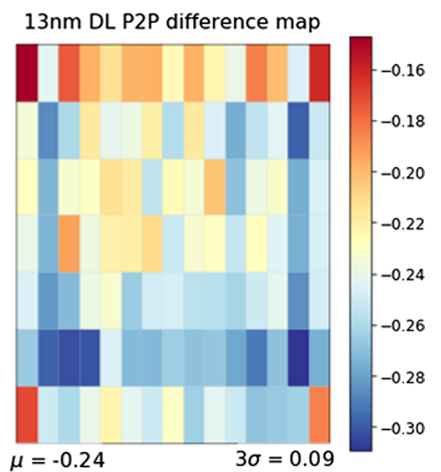

(c)

Fig. 19 13-nm DL intrafield CDU. (a) Measurement with abutted fields in non-CAR metal-oxide resist, (b) measurement with 0.25-mm spacing between fields, (c) delta CD map, showing minor edge/corner impact of EUV + DUV from hybrid BB.

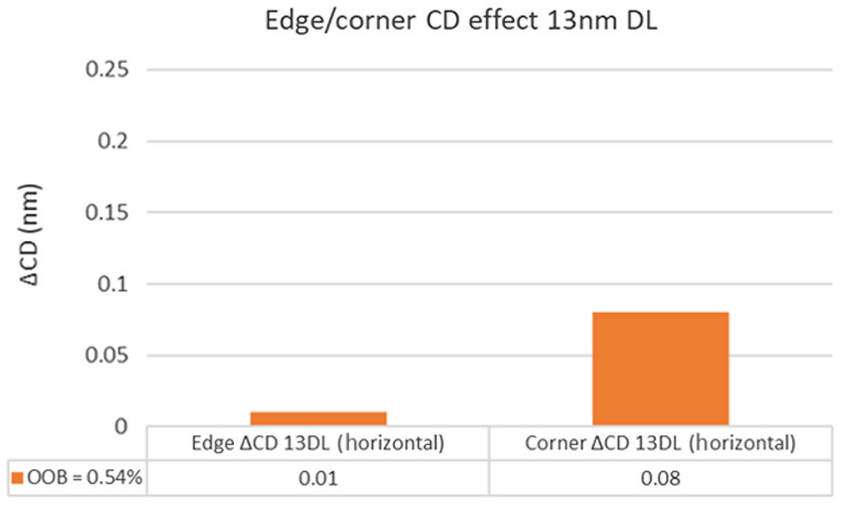

Fig. 20 Edge and corner CD-impact summary on 13-nm DL.

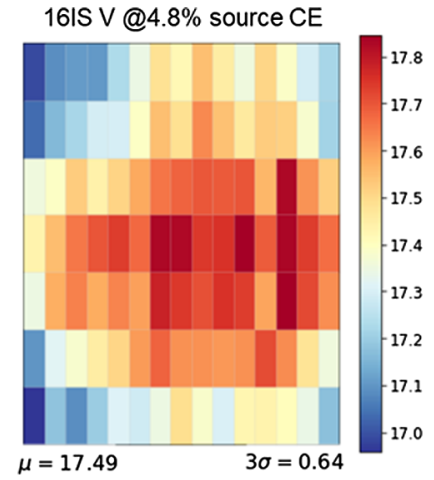

(a)

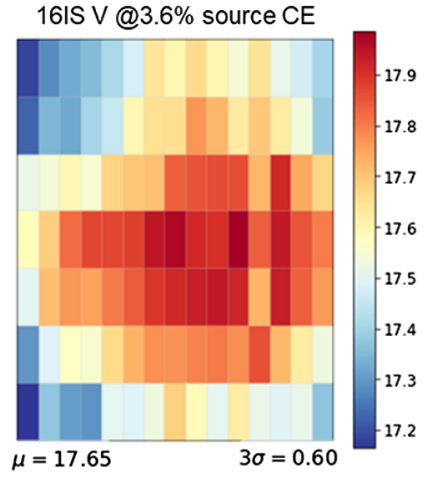

(b)

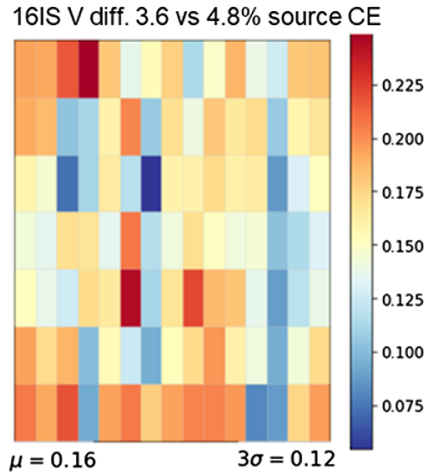

(c)

Fig. 21 16-nm IS with abutted fields intrafield CDU. (a) Measurement with $4.8 \%$ source CE and $0.37 \%$ DUV/EUV in EUVJ-3030, (b) measurement with $3.6 \%$ source CE and $0.54 \%$ DUV/EUV in EUVJ-3030, and (c) delta CD map

also does not show a measurable edge/corner effect in CD-delta. Apparently, the CAR resist used for the 16-nm IS is less sensitive to the spurious EUV reflectivity of the hybrid BB than the non-CAR metal-oxide resist used for the 13-nm DL in Figs. 17 and 19.

These results show that the combined edge/corner effects of the NXE:3400B are already well within the limits for $\mathrm{N} 5$ and even $\mathrm{N} 3$ nodes. It should be noted that the test reticle used has a hybrid BB, with $<1 \%$ DUV reflectivity (see Fig. 11). 


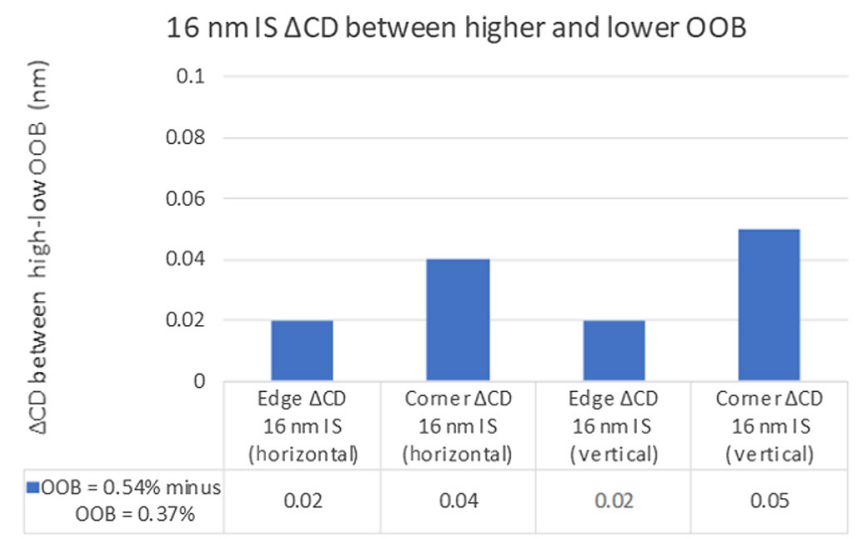

Fig. $22 \mathrm{CD}$-impact on 16-nm IS with abutted fields, difference between OOB $=0.54 \%$ case and $\mathrm{OOB}=0.37 \%$ case.

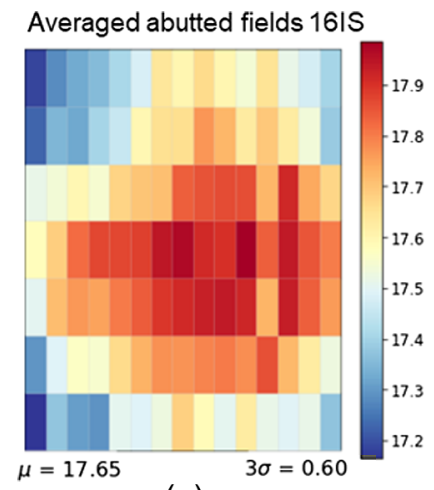

(a)

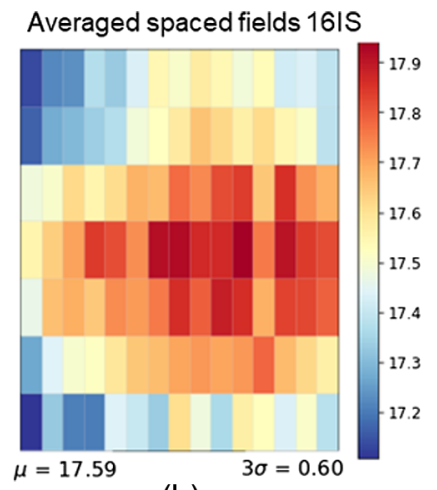

(b)

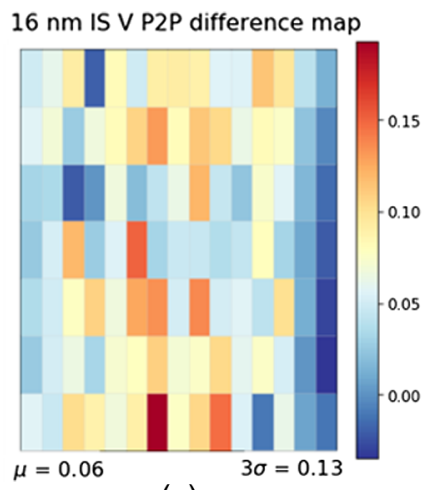

(c)

Fig. 23 16-nm IS intrafield CDU, in CAR resist. (a) Measurement with abutted fields, (b) measurement with $0.25-\mathrm{mm}$ spacing between fields, and (c) delta CD map, showing no measurable edge/corner impact of EUV+DUV from hybrid BB.

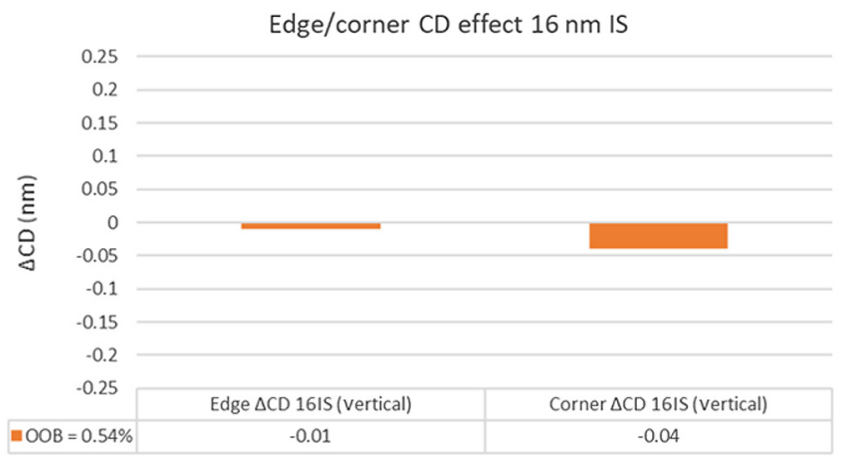

Fig. 24 Edge and corner CD-impact summary on 16-nm IS.

\section{Summary and Conclusions}

The measured source emission spectra from high-power EUV machines are presented. The PP to droplet distance in $Z$ direction is identified as a possible control knob for EUV FB spectrum from source plasma. Rigorous imaging simulation results using different measured EUV spectra showed that the EUV spectrum variation does not have a significant impact on imaging performance. This can be understood by the strong spectral filtering of the multiple ML mirrors of the 
optical column. This shows that there is no need to overconstrain EUV source with additional requirements on EUV FB spectrum and that the EUV imaging performance is robust against variations in $\mathrm{CE}$ and targeting.

The impact of optical column and reticle BB on imaging performance is discussed. Imaging experimental results with/without pellicle and with/without DGLm show that the DGLm is very effective in DUV suppression at wafer level, even if in most measured cases it is not needed. In general, the spectral purity performance of NXE:3400 systems is shown to be well within the specifications for N5 node and allows extension to higher source powers and to future nodes. In fact, higher source powers will have higher CE, which promises even lower DUV/EUV ratio.

It has been demonstrated that the induced variations in DUV level do not affect imaging performance to any significant degree. It should be noted though that less effective BB designs and more sensitive resists might still show an edge/corner CD-shift due to DUV and/or EUV.

Additionally, we demonstrated a practical method of establishing both DUV and EUV CDsensitivities for customer masks and processes in the field, by in-line changing the DUV/EUV ratio by varying the PP focus to droplet distance in $Z$ direction in the LPP source.

\section{Acknowledgments}

The authors would like to thank Wouter Varenkamp, Kees Ricken, Cheuk-Wah Man, Damien Aussems, Jochem Huijs, Hilbert van Loo, Zhaohan He, and Francesco Torretti for the help with measurements and analysis, as well as Igor Fomenkov, Guido Schiffelers, Felix Wählisch, Eelco van Setten, Alex Schafgans, Peter Mayer, Evan Davis, Oscar Versolato, Wim van der Zande, Fred Bijkerk, and Laurens de Winter for fruitful technical discussions. We would like to thank John McNamara, Peter Havermans, Timon Fliervoet, Rene Carpaij, Joseph Zekry, and Joost Gielis at ASML for the support for this project. The transmission grating spectrometer has been supported by the FOM Valorization Prize awarded to F. Bijkerk in 2011 and the NanoNextNL Valorization Grant awarded to M. Bayraktar in 2015. The paper is a revision and update of our SPIE Proceedings paper presented earlier this year. ${ }^{24}$

\section{References}

1. M. van de Kerkhof et al., "Lithography for now and the future," Solid-State Electron. 155, 20-26 (2019).

2. M. van de Kerkhof et al., "Understanding EUV-induced plasma and application to particle contamination control in EUV scanners," Proc. SPIE 11323, 113230Y (2020).

3. N. Davydova et al., "Impact of an etched EUV mask black border on imaging: part II," Proc. SPIE 8880, 888027 (2013).

4. M. van de Kerkhof et al., "Enabling sub-10 nm node lithography: presenting the NXE:3400B EUV scanner," Proc. SPIE 10143, 101430D (2017).

5. A. Heays et al., "Photodissociation and photoionisation of atoms and molecules of astrophysical interest," A\&A 602, A105 (2017).

6. I. Fomenkov et al., "Light sources for high-volume manufacturing EUV lithography: technology, performance, and power scaling," Adv. Opt. Technol. 6(3-4), 173-186 (2017).

7. G. Wannier, Statistical Physics, Dover Publications, New York (1987).

8. H. Nishimura et al., "Development of extreme-ultraviolet light source by laser-produced plasma," Rev. Laser Eng. 36(APLS), 1125-1128 (2008).

9. I. Fomenkov, EUV Source Workshop, edited by V. Bakshi (2018).

10. H. Sakaguchi et al., "Absolute evaluation of out-of-band radiation from laser-produced tin plasmas for extreme ultraviolet lithography," Appl. Phys. Lett. 92(11), 111503 (2008).

11. F. Torretti et al., "Spectral characterization of an industrial EUV light source for nanolithography," J. Phys. D: Appl. Phys. 53, 055204 (2020).

12. CXRO Database, http://henke.lbl.gov/optical_constants/.

13. N. Davydova et al., "EUVL mask performance and optimization," Proc. SPIE 8352, 835208 (2012).

14. M. Bayraktar et al., "Broadband transmission grating spectrometer for measuring the emission spectrum of EUV sources," NEVAC blad 54(1), 14-19 (2016). 
15. E. Davis, ASML Technology Development, private communication (2019).

16. G. Lorusso et al., "Deep ultraviolet out-of-band contribution in extreme ultraviolet lithography: predictions and experiments," Proc. SPIE 7969, 796920 (2011).

17. M. Bayraktar et al., "Broadband spectral characterization of EUV light sources with a transmission grating spectrometer," in (S85), EUV Source Workshop, Dublin (2017).

18. F. Liu et al., "Lithography machine in-line broadband spectrum metrology," in (P43), EUVL Workshop, Berkeley (2019).

19. F. Timmermans et al., "Alternative mask materials for low-k1 EUV imaging," Proc. SPIE 11177, 111470D (2019).

20. G. Watanabe et al., "EUV mask with advanced hybrid black border suppressing EUV and DUV OOB light reflection," Proc. SPIE 10807, 108070N (2018).

21. C. Turley, "EUV mask black border evolution," Proc. SPIE 9235, 923513 (2014).

22. J. van Schoot et al., "High-NA EUV lithography exposure tool progress: program progress and mask impact (Conference Presentation)," Proc. SPIE 10957, 1114710 (2019).

23. D. Brouns et al., "ASML NXE pellicle update," Proc. SPIE 11178, 1117806 (2019).

24. M. van de Kerkhof et al., "Spectral purity performance of high-power EUV systems," Proc. SPIE 11323, 1132321 (2020).

Mark van de Kerkhof began his career at ODME, developing then-novel DVD mastering, and later worked on deep-UV and immersion technologies for Blu-Ray. In 1999, he joined ASML as a senior designer, working on miscellaneous projects for both DUV and EUV scanners, and was responsible for the technical definition and integration of the NXE:3400B EUV scanner as product system engineer. He is currently responsible for EUV scanner plasma technology. He co-authored more than 30 scientific papers and holds more than 70 USA patents.

Biographies of the other authors are not available. 